\title{
Erratum to: Flood exposure and settlement expansion since pre-industrial times in 1850 until 2011 in north Bavaria, Germany
}

\author{
Andrea Früh-Müller • Martin Wegmann • \\ Thomas Koellner
}

Published online: 31 July 2014

(C) Springer-Verlag Berlin Heidelberg 2014

\section{Erratum to: Reg Environ Change}

DOI 10.1007/s10113-014-0633-9

The original version of this article unfortunately contained a mistake. The affiliation of Prof. T. Koellner is incorrect. The correct affiliation is: Professorship of Ecological Services, University of Bayreuth, Universitätsstrasse 30, 95440 Bayreuth, Germany.

The online version of the original article can be found under doi:10.1007/s10113-014-0633-9.

A. Früh-Müller · T. Koellner

Professorship of Ecological Services, University of Bayreuth, Universitätsstrasse 30, 95440 Bayreuth, Germany

e-mail: thomas.koellner@uni-bayreuth.de

A. Früh-Müller $(\bowtie)$

Department of Animal Ecology, Justus-Liebig University, Heinrich-Buff Ring 26-32, 35392 Giessen, Germany

e-mail: andrea.frueh-mueller@bio.uni-giessen.de

M. Wegmann

Department of Remote Sensing, University of Würzburg, Campus Hubland Nord 86, 97074 Würzburg, Germany

e-mail: martin.wegmann@uni-wuerzburg.de 